\title{
In silico Characterization, Molecular Dynamics Simulation and Expression Analysis of Two Class I Chitinases from Aloe barbadensis
}

\author{
Manish Kumar Suthar*, V. Thondaiman, Akula Chinapolaiah, \\ P. Manivel and Manish Kumar Mittal
}
ICAR-Directorate of Medicinal and Aromatic Plants Research, Boriavi, Anand, Gujarat-387310, India

*Corresponding author

\section{A B S T R A C T}

\begin{tabular}{|l|}
\hline Ke y w o r d s \\
Aloe, Chitinase, \\
MD simulation, \\
Docking, \\
Expression \\
\hline Article Info \\
\hline $\begin{array}{l}\text { Accepted: } \\
17 \text { November } 2019 \\
\text { Available Online: } \\
10 \text { December } 2019\end{array}$ \\
\hline
\end{tabular}

\section{Introduction}

Plants produce diverse array of pathogenesisrelated (PR) proteins to protect against attacks of external pathogens. Chitinases are PR proteins that hydrolyze chitin, a homopolymer of N-acetyl D-glucosamine. This polysaccharide is structural component of fungi, insects, crustaceans and green algae (Santos et al., 2004). Plant chitinases belong to glycoside hydrolase 18 and 19 families on the basis of their catalytic mechanism. They are further classified in to seven classes (Class I-VII) based on different amino acid residues forming domains and motifs (Neuhaus et al., 1996; Singh et al., 2007; Ahmed et al., 2012). 
By contrast, the catalytic mechanism of GH19 is not wholly understood. These proteins are constitutively present in leaves, roots, stems and flowers but the high expression of their genes have been reported during external elicitations (Libantova et al., 2009; Porat et al., 2001; Gerhardt et al., 2004). These proteins are of huge significance for their diverse nature and immense potential uses viz. biocontrol of plant pathogenic fungi and insects, biopesticides, production of chitooligosaccharides, single protein production etc. (Islam and Datta 2015). Various organisms produce a wide variety of chitinases that show biochemical as well as biophysical characteristics for different potential uses.

The genus Aloe (family Asphodelaceae) comprises around 360 species. Out of which Aloe barbadensis Miller, A. arborescence, A. ferox and $A$. perryi are known to exhibit medicinal properties. A. barbadensis, in particularly, is well known tropical succulent plant that has been known and grown for their immense pharmaceutical importance since thousands of years(Pugh et al., 2001; Chen et al., 2012). Bioactive constituents from $A$. barbadensis have been known for their antiulcer, anti-hyperglycemic, skin burn healing, anti-asthmatic, antioxidant, hepetoprotective, manti-tumer, antihypercholestrolemic, and anti-inflammatory properties (Tarameshloo et al., 2012; Langmead et al., 2004; Chandan et al., 2007; Lin et al., 2006; Borrelli and Izzo 2000; Kumar et al., 2013; Huseini et al., 2012; Qadir, 2009; Mikadi and Damak, 2008). Despite huge potential applications very little genomic, proteomic and gene content related information is available for A. barbadensis. Such limitations halt bio-prospecting at molecular or gene level of this plant. Present study is the first report on identification and computational characterization of a chitinase from A. barbadensis. Putative chitinase transcripts were identified in raw TSA database. In-silico characterization of respective proteins of transcript sequences and their expression analysis were carried out. The sequence information of putative $A$. barbadensis chitinases were used to generate protein homology models. Further, docking with ligand and molecular dynamics simulations were carried out for $A$. barbadensis chitinase.

\section{Materials and Methods}

\section{Identification of chitinase sequences}

Chitinase [Hordeum vulgare: AAA56787.1] sequence was used for tBLASTn against TSA (Transcriptome Shotgun Assembly) database of Aloe barbadensis. Two raw sequences were retrieved based on score, query cover and length of unigenes. The ORF regions of all unigenes were determined using ExPASy tool (https://web.expasy.org/translate/). Protein sequence obtained from translate tool were again subjected to BLASTp against nonredundant database for proteins at NCBI to identify or to verify the ORF regions. Similarity percentage between identified sequences was determined by Clustal-omega (https://www.ebi.ac.uk/Tools/msa/clustalo/). MEGA6 was used to multiple sequence alignment and phylogenetic analysis of homologs (Tamura et al., 2013). For phylogenetic analysis Neighbor-joining (NJ) approach with bootstrap replicates (1000) was employed.

Gene expression analysis of chitinases in different aloe genotypes

Expression profile of Abchit genes was studied in three genotypes using Real-time PCR detection system and SYBR green PCR master mix. Gene specific primers were designed and GAPDH gene primers were designed as reported earlier (Choudhari et al., 2018). Leaf samples were collected from three 
different genotypes of Aloe barbadensis maintained at DMAPR field. Young leaves were selected to analyze the expression level of two chitinases. These Aloe genotypes have distinct morphological traits. Gel portion of Aloe leaves were removed and remaining samples were used for total RNA isolation. Total RNA from leaves was isolated using GeneJET Plant RNA Purification Mini Kit (Thermo Scientific). A first strand cDNA was prepared using $5 \mu \mathrm{g}$ of total RNA, $500 \mathrm{ng}$ oligo-(dT) 18 primer (supplied with kit) and M-MuLV Reverse Transcriptase supplied with First Strand cDNA Synthesis Kit (Thermo Scientific). For each primer set, a control reaction was also included without template DNA. Real time analysis was conducted using SYBR green master mix (Maxima SYBR Green).Fold change in gene expression was calculated as $2^{-\Delta \Delta \mathrm{Ct}}$ using $\Delta \mathrm{Ct}$ values.

\section{Domains identification and estimation of biochemical parameters}

Protein sequences were deposited at PROSITE expasy (https://prosite.expasy.org) and SMART (http://smart.embl-heidelberg.de) to identify domain structures. Protein properties such molecular weight, isoelectric point, grand average of hydropathicity were predicted using Protparam tool (https://web.expasy.org/protparam/).

\section{Homology modeling}

The protein sequences of Abchit 1 and 2 were used for Blastp search tool against PDB database and four different protein crystal structures were collected based on e-values for closest homology identification. These PDB structures used as templates for model building. Modeller 9.22 was used to develop homology models of Abchit1 and 2. Above five PDB templates were used in Modeller and best template was identified based on distance matrix generated by Modeller and crystallographic resolutions. Modeller generated 50 initial models and based on their DOPE score and GA score best three dimensional model was selected for further analysis. Models were visualized by PyMol and Discovery studio programs.

\section{Docking analysis}

A. barbadensis chitinases were docked with NAG oligomer using Autodock Vina program(Trott and Olson 2010). Structures of A. barbadensis chitinases and ligand were converted in to PDB format using Discovery studio. Docked protein complexes were visualized and analyzed using Discovery studio and PyMol program.

\section{Simulation}

A. barbadensis chitinase models were subjected to $50 \mathrm{~ns}$ MD simulation using GROMACS package(Berendsen et al., 1995). The topology files were generated using GROMOS 96 force field. In preparative steps, The Abchit 1 and 2 proteins were immersed in a box of explicit simple point charge water (SPC water). The dimension of box was kept 1 $\mathrm{nm}$. To yield electrically neutral system $\mathrm{Na}$ or $\mathrm{Cl}$ ions were added. The solvated ent-CPSs were energy minimized by steepest descent methods to remove close van der Waals contacts. The system was equilibrated under NVT and NPT ensemble with position restraint simulations for $500 \mathrm{ps}$ and finally production MD for $50 \mathrm{~ns}$ (constant temperature $300 \mathrm{~K}$ and pressure 1 bar) in triplicates. All the bonds were constrained using LINC algorithm and electrostatic interactions were calculated using Particle Mesh Ewald (PME). Simulations were repeated three times showing high degree of reproducibility. All trajectories like root mean square deviations (RMSD) and root mean square fluctuations (RMSF) were explored by using GROMACS. The DSSP tool was 
employed to analyze secondary structure changes.

\section{Results and Discussion}

Identification of $A$. barbadensis chitinases and validation of their expression in various genotypes

Two putative chitinase sequences were identified from raw TSA database. Both sequences showed 89 percent query coverage with Hordeum chitinase (used as template for search) but showed 61 and 58 percent sequence identities. These chitinases were named as Abchit1 and Abchit2 which have 315 and 319 amino acid residues respectively as suggested by Expasy translation tool. Figure 1 shows the phylogenetic analysis of Abchit1 and Abchit2. Furthermore, based on Clustal-Omega analysis these two homologs show 75.56 percent sequence similarity with each other Figure 2A. It means there are subtle sequence differences between these two chitinases. Phylogenetic analysis indicated that Abchit 1 is more similar to Alium sativum chitinase whereas Abchit2 falls in different evolutionary group (Fig. 1). Overall results showed that Abchit2 probably has evolved as distinct chitinases member. Different classes of plant chitinases show differences in domain organizations. Broadly, all plant chitinases have been classified in four classes. Class I chitinases have $\mathrm{N}$-terminal leader sequence, a cysteine rich chitin binding domain, a hinge region and catalytic main domain. Class II chitinases show significant similarity with Class I chitinases but completely lack the chitin binding domain and the hinge region. Interestingly, Class III chitinases do not show significant similarity with above class I and II enzymes [25] (Collinge et al., 1993). Finally, Class IV chitinases have domain organization similar to class I chitinases but the length of Class IV members is shorter (241-255 aa) than Class I members (about 300 aa). Domain architecture of Abchit1 and Abchit2 is shown in Figure 2B. Abchit1 and Abchit2 have Nterminal leader sequence, Cysteine rich chitin binding domain (CBD) and a main catalytic domain separated by a hinge region from CBD. Figure 3 shows putative di-sulphide bond pattern formation among cysteine residues present in $\mathrm{CBD}$. Based on the length of amino acid residues and domain organization, Abchit1 and Abchit2 fall in Class I of chitinases and various biochemical parameters are shown for these proteins in Table 1. Biochemical parameters show that both Abchit 1 and Abchit2 have Mw around 33 $\mathrm{kDa}$ which is also supposed to be character of class I chitinases (Collinge et al., 1993). By contrast, these chitinases have different physical nature as Abchit1 is acidic in nature whereas Abchit2 shows basic nature as indicated by their respective pI. Plant chitinases have been classified as acidic or basic chitinases (Flach et al., 1992). Thus, within class-I, these chitinases can be grouped in different subclasses of chitinases. Relative expression of Abchit1 and 2 is shown in Figure 4. The results of expression analysis suggested that both of the chitinases are expressed in leaves of different genotypes. However, there are variations in the level of expression among various genotypes for individual chitinases. Our experiment shows that both Abchit1 and 2 are constituently expressed in mesophyll region of leaves.

\section{Homology model and docking analysis of Aloe chitinases}

Initially, blastp tools yielded four PDB structure (2dkv, 4dwx, 4jol and 1cns) based on homology. Finally, 2dkv (class I chitinase of Oryza sativa L. japonica) was selected to use it as a template for homology model for Abchit1 and 2(Kezuka et al., 2010).Both chitinases show 11 helices separated by $\beta$ turns and various di-sulphide bonds. Which were further validated and Ramachandaran 
plot analysis of these proteins are shown in Figure 5. These analyses show almost all amino acid residues in both chitinases are under most favored region and additional allowed regions (Fig. 5 and Table 1). Both the Ramachandaran plot analysis and instability index predicted by ProtParam indicated that these three-dimensional structures are stable. Three dimensional models for Abchit1 and Abchit 2 shows significant similarities.

However, the detailed catalytic mechanism and substrate binding of plant chitinases have not been elucidated yet. Although some crystal structures complex with NAG and NAG oligo have been reported (Ohnuma et al., 2012; Huet et al., 2008). Chitin oligosaccharides instead of NAG monomer as substrate to complex with chitinase crystal studies have appeared better way to understand substrate binding (Ohnuma et al., 2012). Hence, in present study NAG oligomer was used for docking. Docking of Abchit1 and Abchit2 with $\mathrm{N}$-acetyl glusamine oligomer is shown in Figure 6. AbChit1 and Abchit2 have two lobes rich in $\alpha$-helical structures. Substrate binding site is located between these lobes. Figure 7 shows the amino acid residues forming contacts with substrate. Similar substrate binding was reported in chitinase from Oryza sativum (Kezuka et al., 2010).

Various loops are extended at both end of substrate binding groove.NAG binds at substrate binding cleft similar to shown in Oryza sativum chitinases (Kezuka et al., 2010). Also, the structural fluctuations of loop regions have shown their participation in catalytic mechanisms of chitinases. These are the structural features of G19 chitinases found in plants (Ohnuma et al., 2012).

\section{MD simulation}

Three dimensional models of Abchit1 and Abchit2were analyzed by 50 ns MD simulation. Figure 8A shows RMSD analysis for Abchit1 and Abchit2. After initial increase RMSD for both chitinases achieved a stationary phase after 5 ns of simulation. RMSD of Abchit 2 show some increase after $12 \mathrm{~ns}$ of simulation but on later stages of simulations both of proteins showed steady phase as indicated by RMSD values indicating stability of modeled proteins. Figure $8 \mathrm{~B}$ shows the radius of gyration ( $\mathrm{Rg}$ ) of chitinases during MD simulation. This radius of gyration is an indication of compactness of proteins. $\mathrm{Rg}$ analysis of Abchit1showed steady states after 20 ns indicating that protein structure are compact having stable folding structure. Whereas, Rg of Abchit 2 showed initial steady state up to $25 \mathrm{~ns}$ and then a small decrease up to $30 \mathrm{~ns}$ followed by steady state. Overall $\mathrm{Rg}$ analysis suggested that Abchit2 showed some conformational changes during simulation but Abchit1 showed comparatively stable conformation. Further, RMSF analysis will help to understand this fluctuation over MD simulation. Figure 9 showed comparative fluctuations of amino acid residues of both chitinases. RMSF analysis showed significant overlapping in comparative RMSF of both chitinases. But, Abchit2 showed more conformational fluctuations as compared to Abchit1.Overall, the main helical content showed less fluctuations and remain stable during entire simulation. Figure 10A and 10B show secondary structure analysis of Abchit1 and Abchit2 during MD simulation. These analyses indicated that over simulation time overall secondary structure of both chitinases remained unaltered. 
Table.1 Biochemical parameters and Ramachandran plot analysis of Abchit1 and Abchit2

\begin{tabular}{|c|c|c|}
\hline & Abchit1 & Abchit2 \\
\hline Amino acids & 315 & 319 \\
\hline Molecular weight (kDa) & 32.72 & 33.58 \\
\hline pI & 5.03 & 7.40 \\
\hline Instability Index & 37.94 & 34.78 \\
\hline Aliphatic Index & 58.98 & 62.82 \\
\hline $\begin{array}{c}\text { Grand average of } \\
\text { hydropathicity (GRAVY) }\end{array}$ & -0.191 & -0.202 \\
\hline $\begin{array}{c}\text { Ramchandran Plot analysis } \\
\text { Residues in most favored } \\
\text { region }\end{array}$ & $86.4 \%$ & $86.7 \%$ \\
\hline $\begin{array}{c}\text { Residues in additional } \\
\text { allowed region }\end{array}$ & $12.6 \%$ & $12.8 \%$ \\
\hline
\end{tabular}

Fig.1 Phylogenetic analysis of various chitinases. Phylogenetic tree was generated using MEGA6 prog

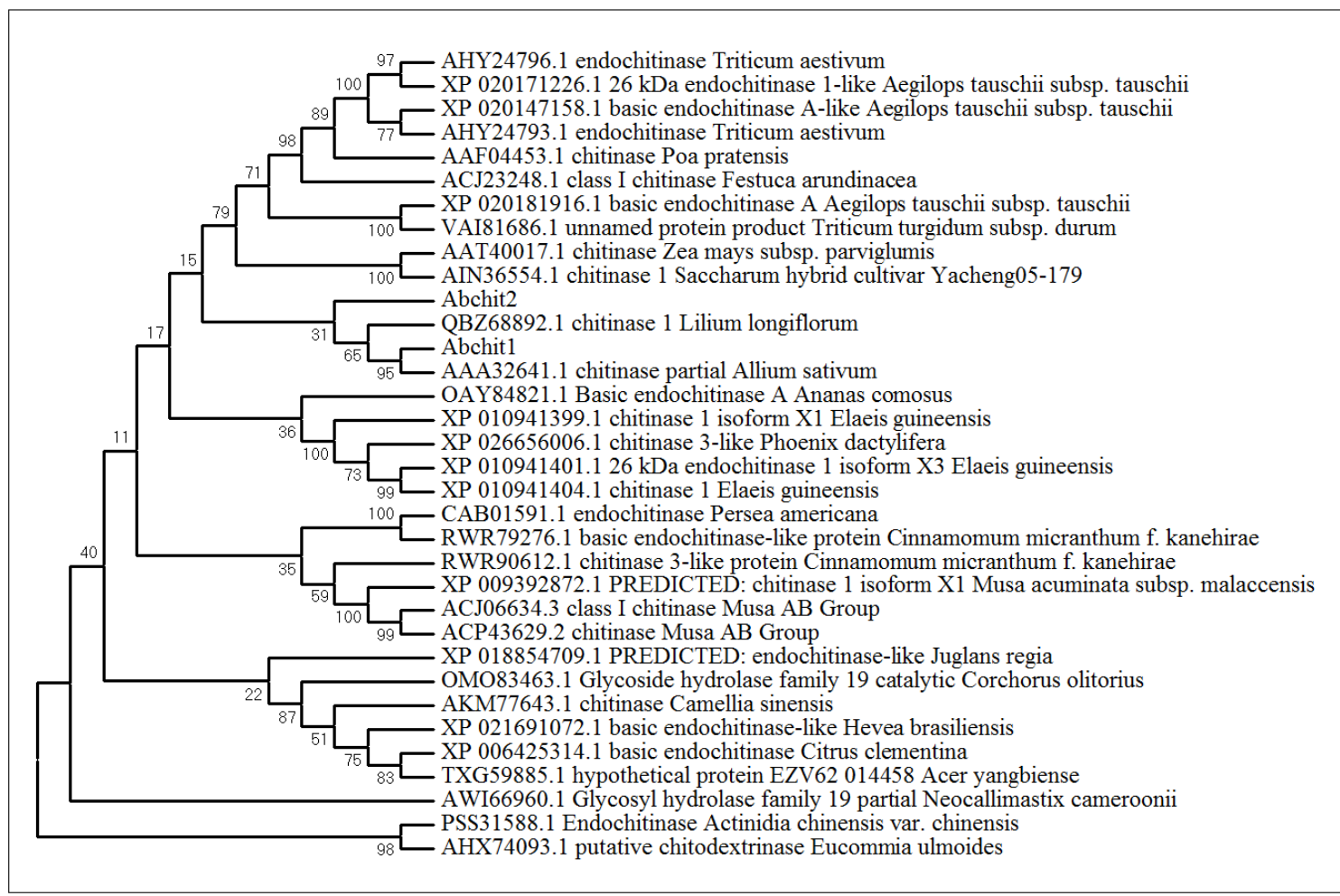


Fig.2 Sequence alignment and domain organization of Abchit1 and Abchit2. A. Clustal-omega based sequence alignment of identified chitinases. B. Domain identification and their arrangement in Abchit 1 and Abchit2 based on SMART and Prosite analysis. N- to C- terminal:

Leader sequence, Cysteine rich Chitin Binding Domain (CBD) and main catalytic domain

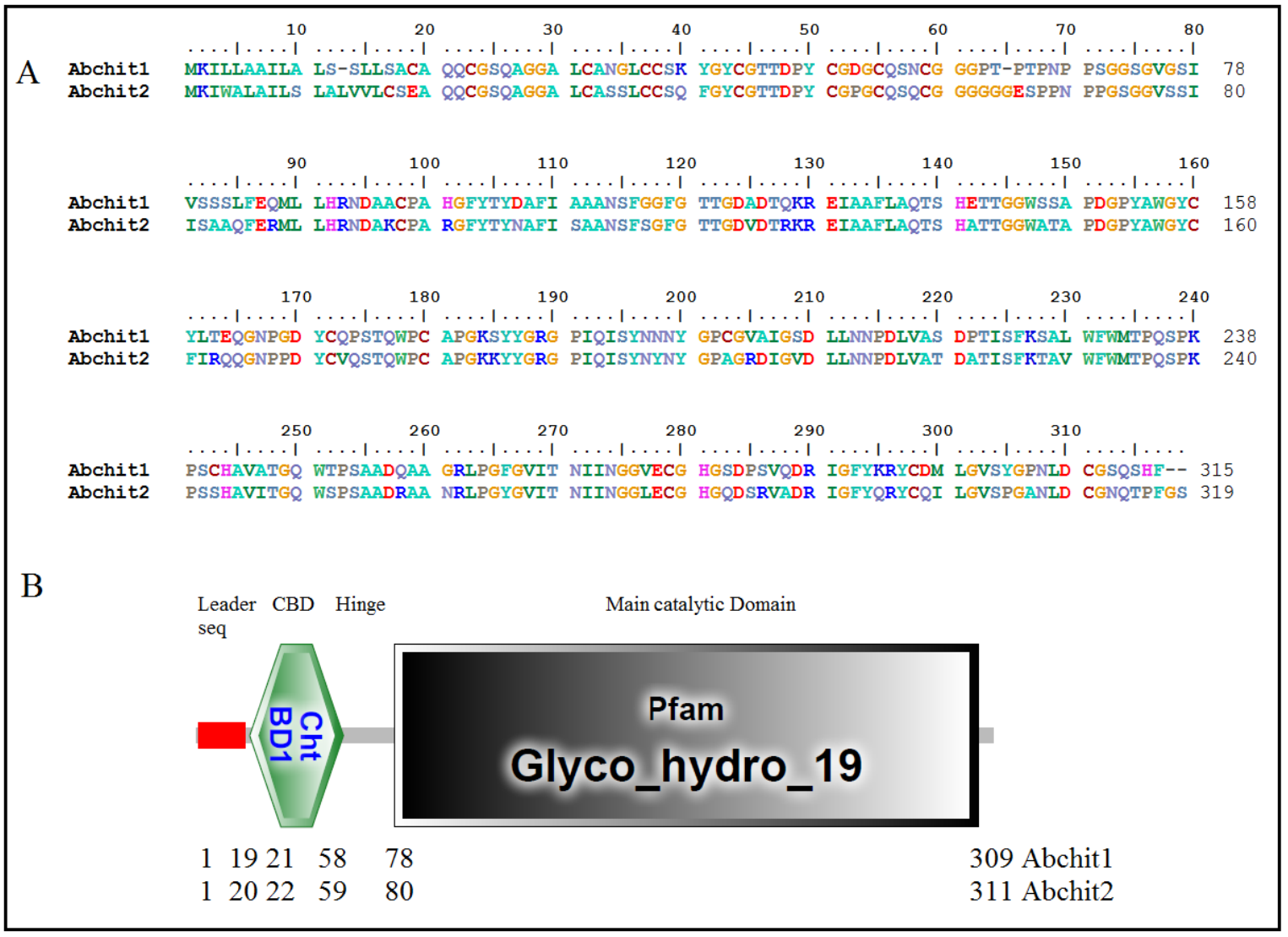

Fig.3 Positions of di-sulphide bond among various Cysteine residues of CBDs in Abchit1 and Abchit 2 as determined by Prosite analysis

AQQCGSQAGGALCASSLCCSQFGYCGTTDPYCGPGCQSQCGG Abchit1 AQQCGSQAGGALCANGLCCSKYGYCGTTDPYCGDGCQSNCGG Abchit2 
Fig.4 Expression profile of Abchit1 and 2 in different genotypes. In each data set 1, 2 and 3 represent different genotypes of Aloe barbadensis

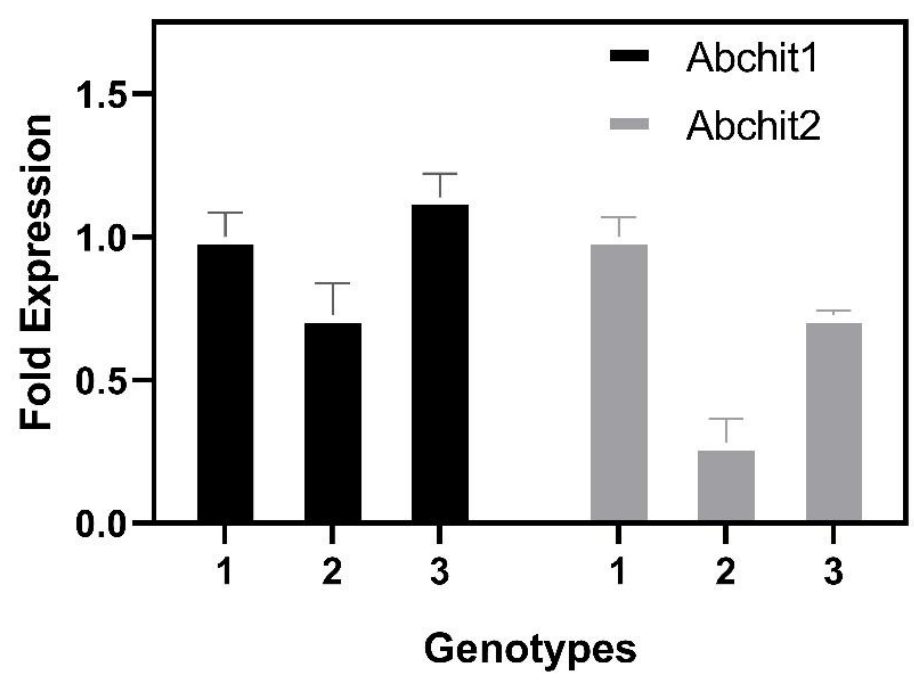

Fig.5 Ramachandran plot analysis of Abchit1 (A) and Abchit2 (B). Plot was generated using 3D models of both chitinases and Discovery studio prog (http://accelrys.com/products/collaborativescience/biovia-discovery-studio/)
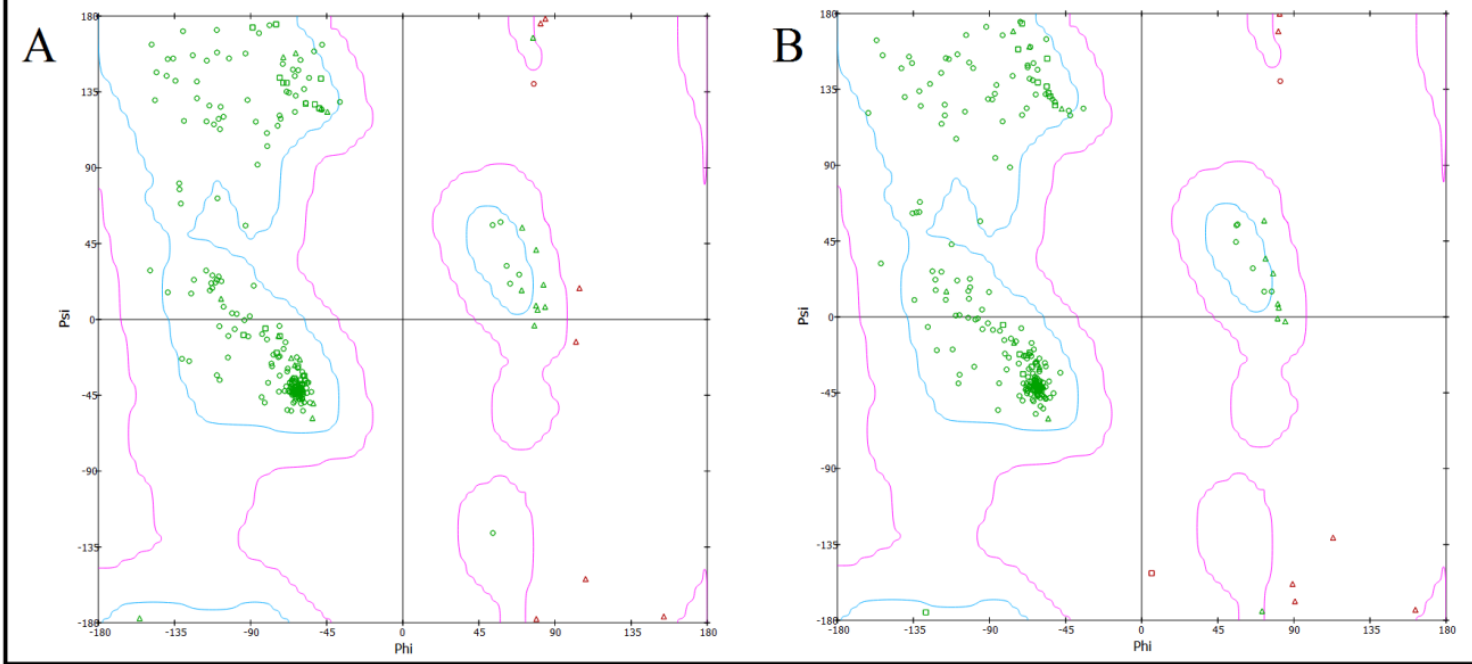
Fig.6 Three dimensional structure of Abchit1 and Abchit2 along with substrate analyzed by PyMol (https://pymol.org/2/).A represents superimposition of both proteins. Abchit1 and Abchit 2 are represented with red and green color respectively. B represents surface structure of Abchit1 and active site region between to lobes of protein

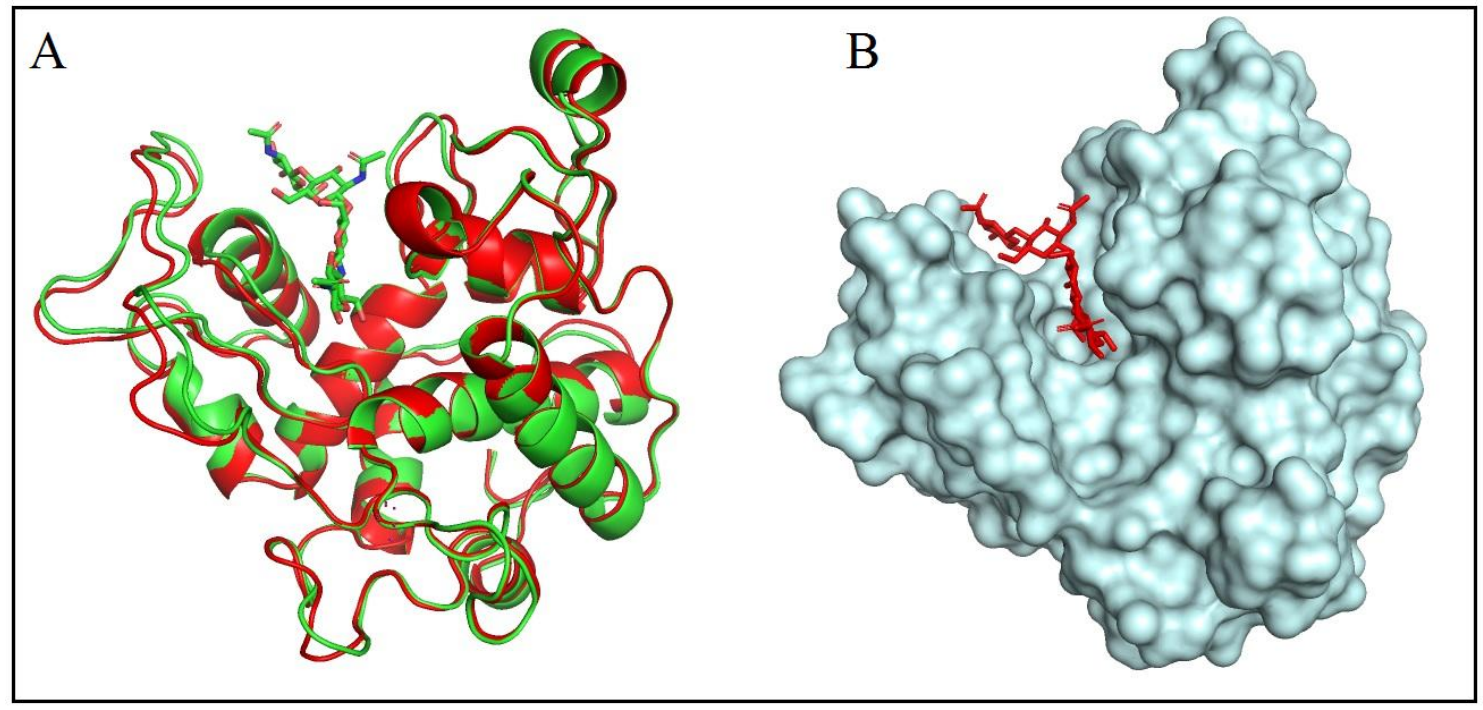

Fig.7 Abchit1 and Abchit2 amino acid residues interacting with oligo NAG substrate. Abchit1 and Abchit2 are represented by red and blue color respectively. Dashed line represents hydrogen bond formation

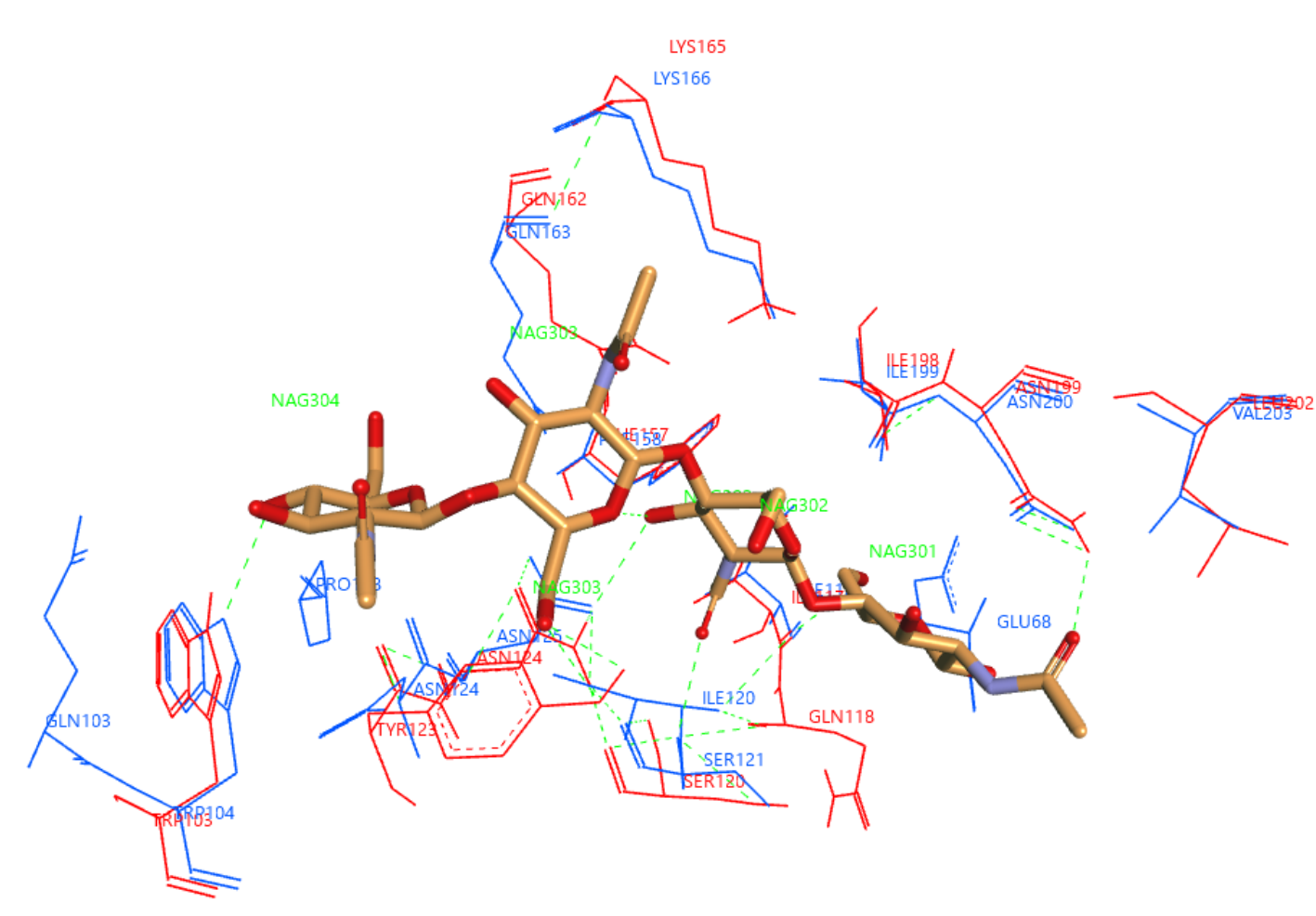


Fig.8 MD simulation: RMSD and radius of gyration: A represents RMSD time course of Abchit1 (black) and Abchit2 (red) during MD simulation. B represents change in Rg of Abchit1 (black) and Abchit2 (red) during MD simulation

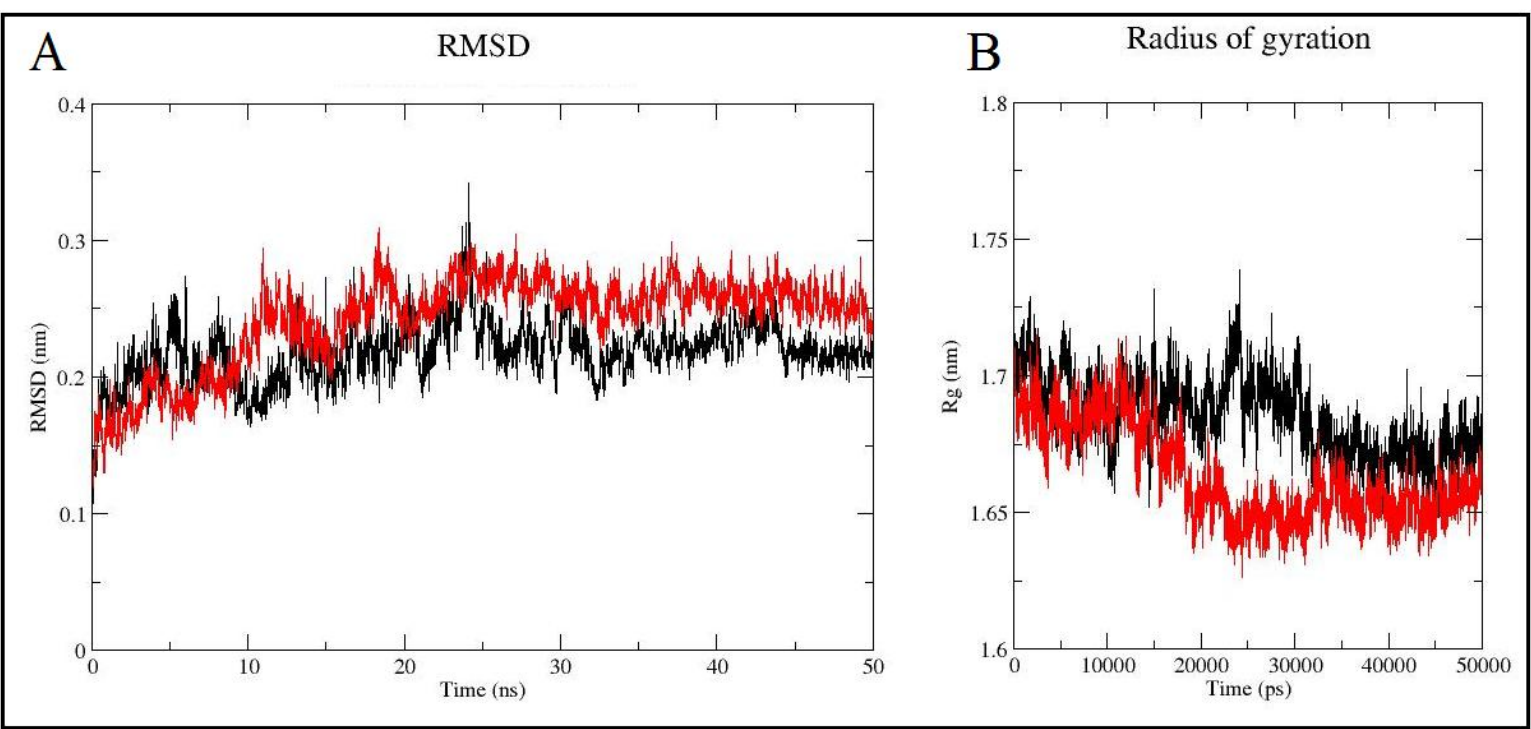

Fig.9 RMSF of Abchit1 (black) and Abchit2 (red) during 50 ns MD simulation. Analysis shows more RMSF in the loop regions of both proteins. Abchit 2 shows more loop fluctuations as compare to Abchit1

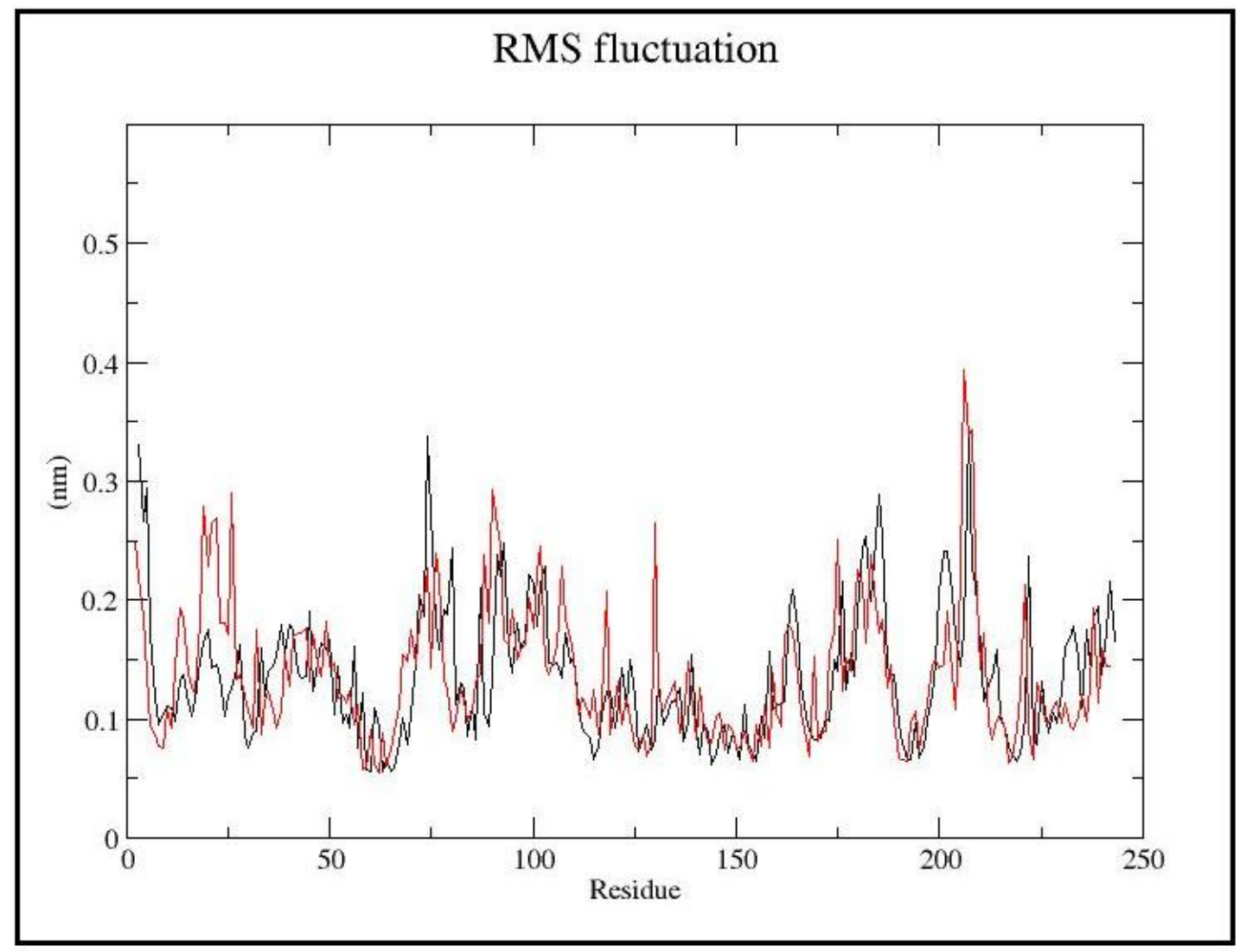


Fig.10 Secondary structure analysis of Abchit1 (A) and Abchit2 (B) during MD simulation. Figure represents total structure change and various structural classed of proteins

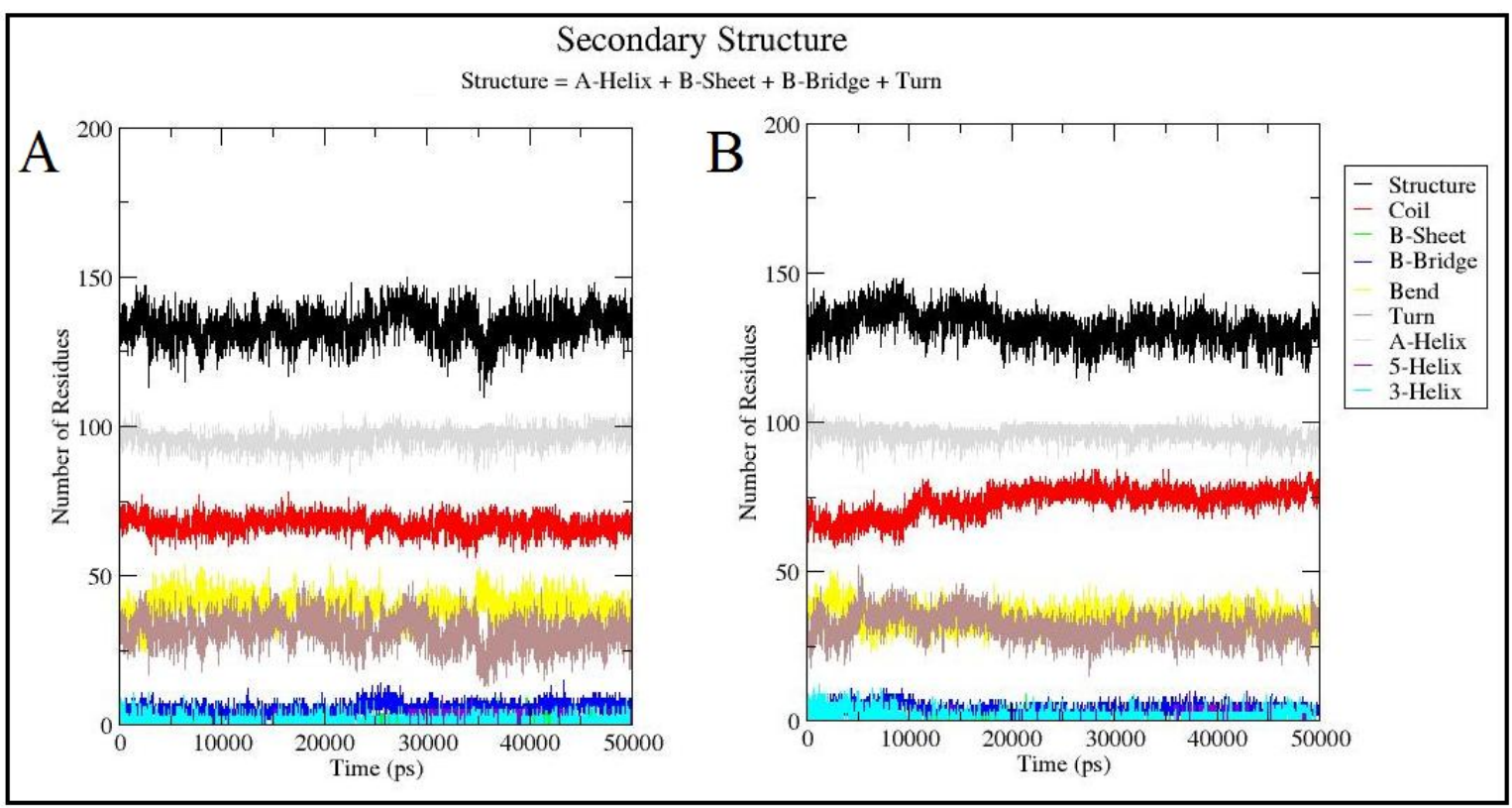

But, number of amino acids participating in structural bends formation showed some alterations. Results of present MD simulation indicated that both chitinases showed stable protein three dimensional structure, constant secondary structure but their loop regions shows some functions which depends upon differences in the primary structures of corresponding loops regions. Present study identify two class - I chitinases from Aloe barbadensis. These proteins show typical structural domain pattern consisting a leader sequence, a cysteine rich CBD domain and a main catalytic domain separated from CBD by a hinge region. The three-dimensional structure of these chitinases shows bi-lobed structure and the substrate binding site is present between these two lobes. Residues forming contacts with substrate were identified in both the chitinases which shows similar pattern of binding with NAG oligomer. MD simulation showed that the three dimensional structure of Abchit1 and Abchit2 is stable but the loop flexibility of Abchit 2 is different from Abchit1. These differences in flexibilities are due to different primary structures of these proteins. Further biochemical analysis of these proteins may lead to development of new biocontrol agents or transgenic plants to cope with plant pathogens.

\section{Acknowledgement}

We thank ICAR-DMAPR, India for the financial support.

\section{References}

Ahmed, N.U., Park, J.I., Seo, M.S., Kumar, T.S., Lee, I.H., Park, B.S. and Nou, I.S. (2012). Identification and expression analysis of chitinase genes related to biotic stress resistance in Brassica. Mol Biol Rep 39:3649-3657.

Berendsen, H.J.C., van der Spoel, D. and van Drunen, R. (1995) GROMACS: A message-passing parallel molecular dynamics implementation. Comp Phys Comm 91: 43-56.

Borrelli, F., and Izzo, A.A. (2000) The plant kingdom as a source of anti-ulcer 
remedies. Phytother Res 14:581-591.

Chandan, B.K., Saxena, A.K., Shukla, S., Sharma, N., Gupta, D.K., Suri, K.A., Suri, J., Bhadauria, M. and Singh, B. (2007) Hepatoprotective potential of Aloe barbadensis mill against carbon tetrachloride induced hepatotoxicity. J Ethnopharmacol 111:560-566.

Chen, W., Van Wyk, B.E., Vermaak, I. and Viljoen, A.M. (2012) Cape aloes-a review of the phytochemistry, pharmacology and commercialisation of Aloe ferox. Phytochem Lett 5:1-2.

Choudhri, P., Rani, M., Sangwan, R.S., Kumar, R., Kumar, A. and Chhokar, V. (2018) De novo sequencing, assembly and characterisation of Aloe vera transcriptome and analysis of expression profiles of genes related to saponin and anthraquinone metabolism. BMC Genomics 19:427.

Collinge, D.B., Kragh, K.M., Mikkelsen, J.D., Nielsen, K.K., Rasmussen, U. and Vad, K. (1993) Plant chitinases. Plant J 3:31-40.

De A Gerhardt, L.B., Magioli,C., Perez, A.B.U.C.M, Margis, R., SachettoMartins, G. and Margis-Pinheiro M. (2004) AtchitIV gene expression is stimulated under abiotic stresses and is spatially and temporally regulated during embryo development. Genet Mol Biol., 27:118-123.

Flach, J., Pilet, P.E. and Jollès, P. (1992) What's new in chitinase research?. Experientia 48:701-716.

Huet, J., Rucktooa, P., Clantin, B., Azarkan, M., Looze, Y., Villeret, V. and Wintjens, R. (2008) X-ray structure of papaya chitinase reveals the substrate binding mode of glycosyl hydrolase family 19 chitinases Biochemistry 47: 8283-8291.

Huseini, H.F., Kianbakht, S., Hajiaghaee, R. and Dabaghian, F.H. (2012) Antihyperglycemic and anti- hypercholesterolemic effects of Aloe vera leaf gel in hyperlipidemic type 2 diabetic patients: a randomized doubleblind placebo-controlled clinical trial. Planta Med 78: 311-316.

Islam, R. and Datta, B. (2015) Diversity of chitinases and their industrial potential. Int J Appl Res 1:55-60.

Kezuka, Y., Kojima, M., Mizuno, R., Suzuki, K., Watanabe, T. and Nonaka, T. (2010) Structure of full-length class I chitinase from rice revealed by $\mathrm{X}$-ray crystallography and small-angle X-ray scattering. Proteins 78:2295-2305.

Kumar, M., Rakesh, S., Nagpal, R., Hemalatha, R., Ramakrishna, A., Sudarshan, V., Ramagoni, R., Shujauddin, M., Verma, V., Kumar, A. and Tiwari, A. (2013) Probiotic lactobacillus rhamnosus GG and Aloe vera gel improve lipid profiles in hypercholesterolemic rats. Nutrition 29:574-9.

Langmead, L., Makins, R.J. and Rampton, D.S. (2004) Anti-inflammatory effects of aloe vera gel in human colorectal mucosa in vitro Aliment. Pharmacol Ther 19:521-527.

Libantová, J., Kämäräinen, T., Moravčíková, J., Matušíková, I. and Salaj, J. (2009) Detection of chitinolytic enzymes with different substrate specificity in tissues of intact sundew (Drosera rotundifolia L). Mol Biol Rep36:851-856.

Lin, J.G., Chen, G.W., Li, T.M., Chouh, S.T., Tan, T.W. and Chung, J.G. (2006) Aloe-emodin induces apoptosis in T24 human bladder cancer cells through the p53 dependent apoptotic pathway. J Urol 175: 343-7.

Miladi, S. and Damak, M. (2008) In vitro antioxidant activities of Aloe vera leaf skin extracts. J Soc Chim Tunisie 10:101-109.

Neuhaus, J.M., Fritig, B., Linthrost, H.J.M., Meins, F., Mikkelsen, J.D. and Ryals J 
(1996) A revised nomenclature for chitinase genes. Plant Mol Biol Rep 14:102-104.

Ohnuma, T., Numata, T., Osawa, T., Inanaga, H., Okazaki, Y., Shinya, S., Kondo, K., Fukuda, T. and Fukamizo, T. (2012) Crystal structure and chitin oligosaccharide-binding mode of a 'loopful' family GH19 chitinase from rye, Secale cereale, seeds FEBS J 279:3639-3651.

Porat, R., Vinokur, V., Holland, D., Gregory McCollum, T. and Droby, S. (2001) Isolation of a citrus chitinase cDNA and characterization of its expression in response to elicitation of fruit pathogen resistance. J Plant physiol 158:1585-1590.

Pugh, N., Ross, S.A., ElSohly, M.A. and Pasco, D.S. (2001) Characterization of Aloeride, a newhigh-molecular-weight polysaccharide from Aloe vera with potent immunostimulatory activity. J Agric Food Chem 49:1030-1034.

Qadir, M.I. (2009) Medicinal and cosmetological importance of Aloe vera. Int J Nat Ther 2: 21-26.

Santos, I.S., Cunha, M.D., Machado, O.L.T. and Gomes, V.M. (2004) A chitinase from Adenanthera pavonina L seeds: purification, characterisation and immunolocalisation. Plant Science 167:1203-1210.

Singh, A., Isaac-Kirubakaran, S. and Sakthivel, N. (2007) Heterologous expression of new antifungal chitinase from wheat. Protein Expres Purif 56:100-109.

Tamura, K., Stecher, G., Peterson, D., Filipski, A. and Kumar, S. (2013) MEGA6: Molecular Evolutionary Genetics Analysis Version 60. Mol Biol Evol 30:2725-2729.

Tarameshloo, M., Norouzian, M., ZareinDolab, S., Dadpay, M., Mohsenifar, J. and Gazor, R. (2012) Aloe vera gel and thyroid hormone cream may improve wound healing in Wistar rats. Anat Cell Biol 45:170-177.

Trott, O. and Olson, AJ. (2010) AutoDock Vina: improving the speed and accuracy of docking with a new scoring function, efficient optimization and multithreading. J Comput Chem 31:455-461.

\section{How to cite this article:}

Manish Kumar Suthar, V. Thondaiman, Akula Chinapolaiah, P. Manivel and Manish Kumar Mittal. 2019. In silico Characterization, Molecular Dynamics Simulation and Expression Analysis of Two Class I Chitinases from Aloe barbadensis. Int.J.Curr.Microbiol.App.Sci. 8(12): 2399-2411. doi: https://doi.org/10.20546/ijcmas.2019.812.282 